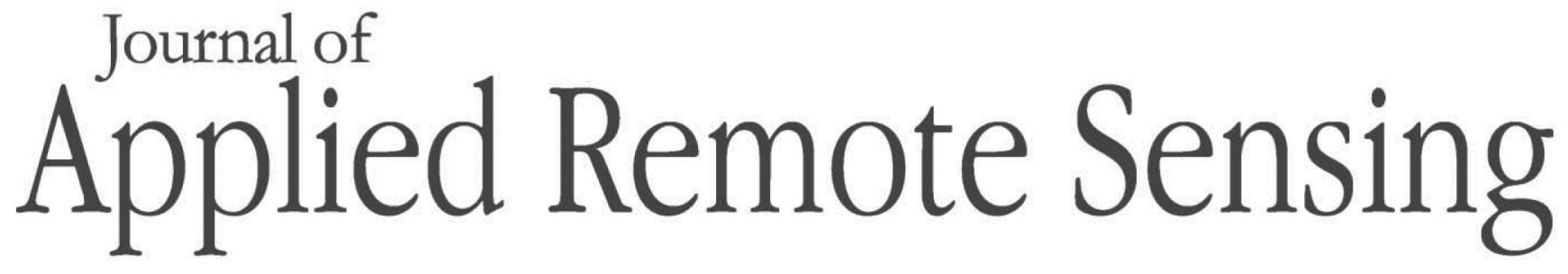

\title{
Towards a critical design of an operational ground segment for an Earth observation mission
}

Tobias Storch

Martin Habermeyer

Sabrina Eberle Helmut Mühle Rupert Müller 


\title{
Towards a critical design of an operational ground segment for an Earth observation mission
}

\author{
Tobias Storch, ${ }^{\text {a }}$ Martin Habermeyer, ${ }^{\text {a }}$ Sabrina Eberle, ${ }^{\text {b }}$ Helmut Mühle, ${ }^{\text {a }}$ \\ and Rupert Müller ${ }^{\mathrm{a}}$ \\ ${ }^{a}$ German Aerospace Center, Earth Observation Center, Münchner Straße 20, \\ 82234 Weßling, Germany \\ tobias.storch@dlr.de \\ ${ }^{\mathrm{b}}$ German Aerospace Center, German Space Operations Center, Münchner Straße 20, \\ 82234 Weßling, Germany
}

\begin{abstract}
The ground segment for the future remote sensing mission Environmental Mapping and Analysis Program (EnMAP; www.enmap.org) is developed by the Earth Observation Center and the German Space Operations Center at the German Aerospace Center. The launch is scheduled for 2017. An operational satellite ground segment is a highly complex heterogeneous system which has to cope with different levels of criticality, novelty, specificity, and to be operated for many years. It consists of equipment, hard- and software as well as operators with their procedures. The strengths of the global coherence of the segment-wide approach bringing these aspects together is examined and not on the local details of segment-specific issues. However, the effects on two software-based elements of the ground segment are considered in more detail, namely the product library and the level 2 geo processor. The development methodology and how the critical design of the complete ground segment finished its detailed design phase successfully was achieved is analyzed. As a measure of the maturity of the design, its stability across the project phases is proposed. (C) 2013 Society of Photo-Optical Instrumentation Engineers (SPIE) [DOI: 10.1117/1.JRS.7.073581]
\end{abstract}

Keywords: image processing; data storage; remote sensing; hyperspectral imaging; architectures; satellites.

Paper 13002 received Jan. 7, 2013; revised manuscript received Feb. 19, 2013; accepted for publication Feb. 21, 2013; published online Mar. 7, 2013.

\section{Introduction}

The evolution towards a critical design for a specific operational satellite ground segment for that of the Environmental Mapping and Analysis Program (EnMAP; www.enmap.org) mission will be studied in detail in the form of a case study. The ground segment for such an Earth observation mission consists of equipment (e.g., antennas, air conditioning), hard- and software (e.g., servers, routers, orbit propagators, image processing software), and operators with their procedures (e.g., flight director, archive operator). The segment-wide approach is considered since it has to cope with a highly complex heterogeneous system which has to be operated for many years with a limited down time for most of its elements. ${ }^{1}$ Therefore, it has to account for, in particular, the different levels of criticality, novelty, and specificity. To illustrate this, two elements of the ground segment are analyzed in more detail which are software-based. The development methodology has to be based on the European Cooperation for Space Standardization (ECSS; www.ecss.nl), which suggests a customized version of the waterfall model lifecycle extended by the V-model. ${ }^{2}$ The design itself is established by an interdisciplinary team, which is also geographically distributed. In order to precise the structure in which the ground segment has to be designed, an overview of the mission and its constituents is given.

0091-3286/2013/\$25.00 @ 2013 SPIE 


\subsection{Mission}

The future satellite mission EnMAP will provide hyperspectral remote sensing image data during its five years of mission operations, which are planned to start in $2017 .{ }^{3}$ The major objectives of the mission are to quantitatively measure and qualitatively analyze diagnostic parameters describing vital processes on the surface of the Earth. The mission provides information about the status of different ecosystems and their response to natural or man-made changes of the environment. The project management is led by the Space Agency at the German Aerospace Center (DLR; www.dlr.de) and the mission is separated into the following three segments. ${ }^{4}$

\subsection{Science (and User) Segment}

The science (and user) segment is led by the German Research Center for Geosciences and together with an advisory group of imaging spectroscopy experts responsible for the definition and maintenance of the scientific objectives and requirements in the form of user requirements. ${ }^{5}$ A science plan details the usage of the data by the international research-oriented user community of science and industry. ${ }^{6}$

Based on the user requirements the mission requirements are constituted by the space (and launch) and ground segment with the project management. These mission requirements are broken down with customer requirements to space (and Launch) segment requirements and ground segment requirements.

\subsection{Space (and Launch) Segment}

The space (and launch) segment is led by Kayser-Threde $\mathrm{GmbH}$, which is also responsible for the hyperspectral instrument realized as pushbroom imaging spectrometers to acquire $232 \mathrm{spec}-$ tral channels in the solar-reflectance range. ${ }^{7}$ The 2-dimensional detector array for the visible and near infrared (VNIR) covers the range from 420 to $1000 \mathrm{~nm}$ and the one for the shortwave infrared (SWIR) covers the range from 900 to $2450 \mathrm{~nm}$ each with 1000 valid pixel in spatial direction (frame) and an analogue-to-digital converter resolution of 14 bits. The rest of the satellite is under the responsibility of Orbitale Hochtechnologie Bremen-System AG. It includes communications in the S-band for telemetry (32 Kbits/s) and telecommand (4 Kbits/s) as well as in the X-band for instrument data (320 Mbits/s), mass memory handling (512 Gbits), measurement and control of orbit and attitude, power generation, and storage (resulting in acquisitions of $167 \mathrm{Kframes/day} \mathrm{of} \mathrm{the} \mathrm{surface} \mathrm{of} \mathrm{the} \mathrm{Earth} \mathrm{plus} \mathrm{calibration} \mathrm{acquisitions} \mathrm{based} \mathrm{on}$ measurements with closed shutter and of the calibration equipment on satellite, Sun, and deep space). The resulting satellite with dimensions $3.1 \times 2.0 \times 1.7 \mathrm{~m}^{3}$ and mass $980 \mathrm{~kg}$ will be launched with the Polar Satellite Launch Vehicle (PSLV) in Sriharikota, India. It will be operated on a polar, Sun-synchronous, morning orbit at $653 \mathrm{~km}$ altitude, which leads to a ground pixel size of approximately $30 \times 30 \mathrm{~m}^{2}$ and thereby in a swath width of $30 \mathrm{~km}$ (and a swath length of $5000 \mathrm{~km} /$ day). The satellite has an across-track tilt capability of \pm 30 deg enabling a revisit time of 4 days and of 21 days within \pm 5 deg.

\subsection{Ground Segment}

The ground segment (GS) is led by the Earth Observation Center (EOC; www.eoc.dlr.de) and the German Space Operations Center (GSOC; www.gsoc.dlr.de) at DLR. ${ }^{8}$ The EOC has long lasting experience with ground-based, airborne, and spaceborne (hyperspectral) data, whereas the GSOC controls and commands several satellite missions. Based on the information of Sec. 1.3, $542 \mathrm{Gbit} /$ day that are loss-less compressed to at most $339 \mathrm{Gbit} /$ day of hyperspectral data as well as $80 \mathrm{Gbit} /$ day for calibration, high-rate housekeeping, and protocol data will have to be transmitted via X-band to EOC multimission ground station in Neustrelitz, Germany. Approximately $30 \mathrm{~min} /$ day of X-band communications are sufficient, which are achieved by four contacts/day. The same holds for S-band contacts to GSOC multimission ground station in Weilheim, Germany, for real time and low-rate housekeeping telemetry transmission, in 
particular for a continuous monitoring of the satellite health, as well as telecommand radiation. These are considerations on the data (and link) budget. All these data are archived.

The various aspects of a satellite ground segment are depicted, which gives a measure for its complexity and motivates the degree of abstraction chosen at the ground segment level. For routine operations the responsibilities of the GS can be illustrated in the context of one of the major technical budgets of the GS, which is the system response time. The system response time is defined as the maximal duration between the placement of an observation request by a user and the delivery of the corresponding product to the user. We consider two requests for observations (request \#1 and request \#2) with corresponding up- and downlinks and one request for long-term archived data (request \#3) all with corresponding processings (see Figs. 1 and 2). The sequence is subdivided at the event the scheduling of the observation requests is performed.

The S- and X-band ground stations communicate their availabilities (shown in blue in Fig. 1) once a week for at least two weeks in the future. Based on this information and the requests for

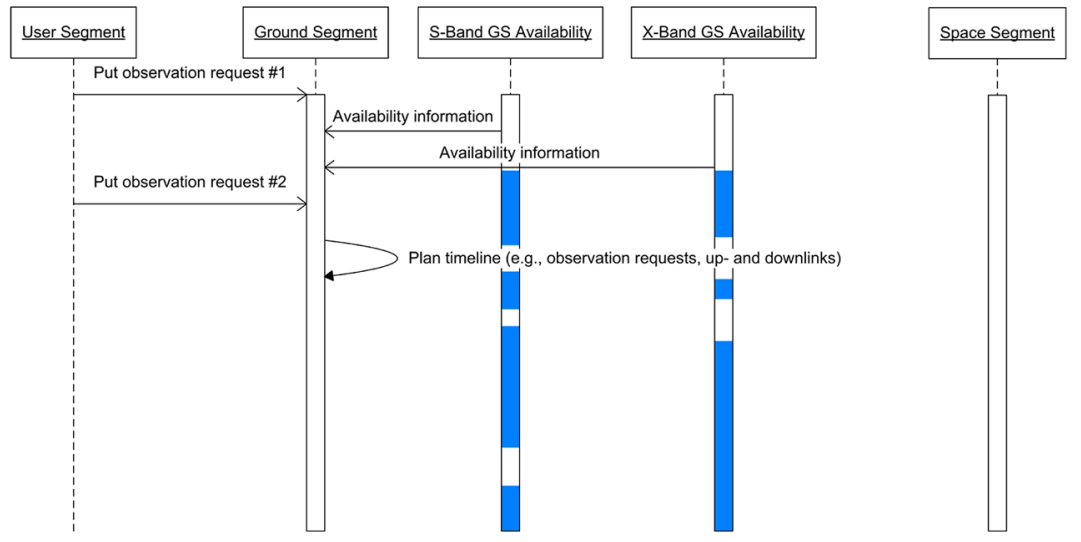

Fig. 1 System response time: part 1 (blue: station contact available, white: station contact not available).

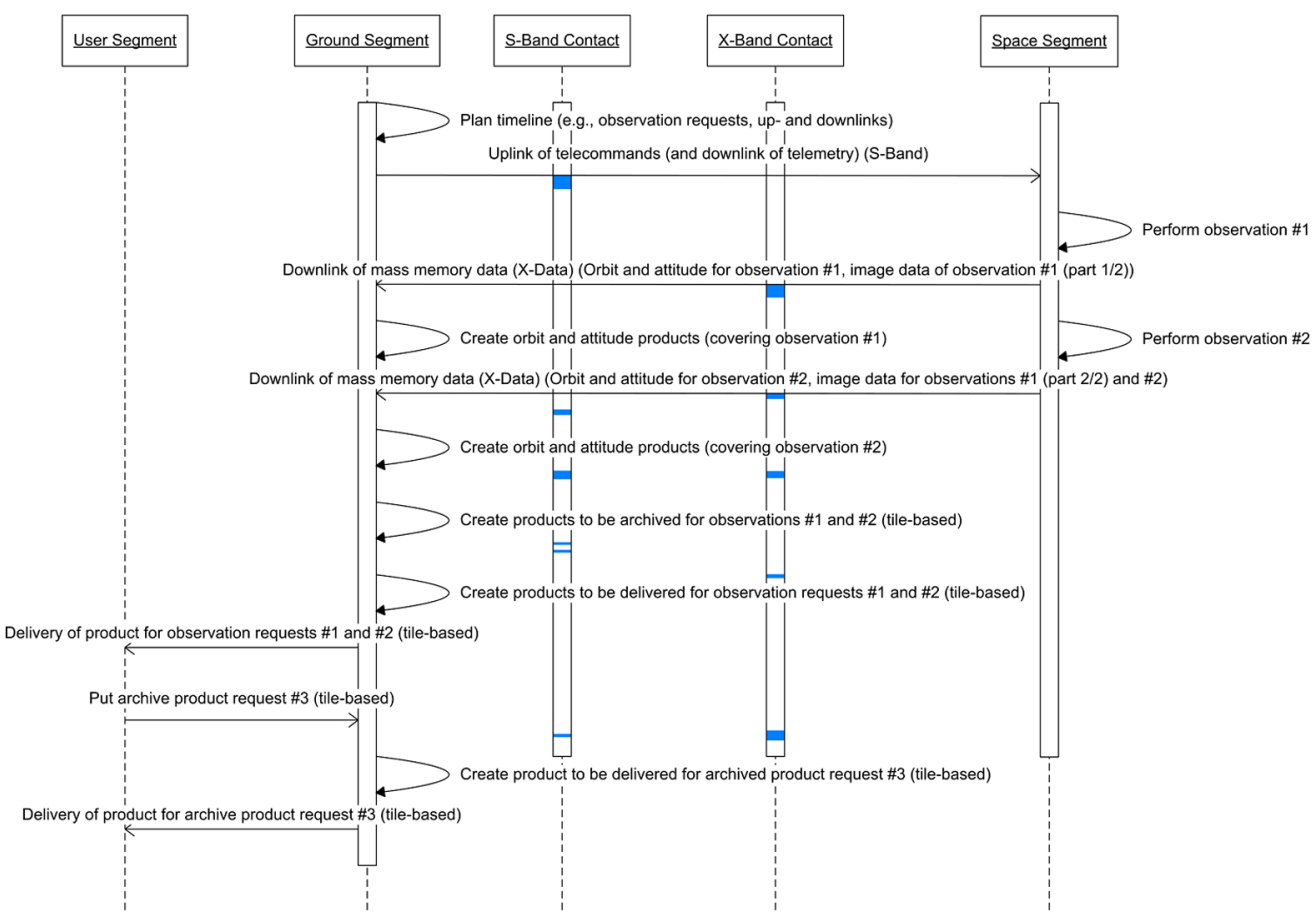

Fig. 2 System response time: part 2 (blue: station contact scheduled, white: station contact not scheduled). 
observations submitted by users, a planning of the subsequent timeline is performed $6 \mathrm{~h}$ before each S-band contact. Users can put requests for observations in the time frame between their registration (request \#1) and at least $7 \mathrm{~h}$ before the S-band contact (request \#2), when the corresponding acquisition has to be commanded. Since at least every $12 \mathrm{~h}$ an S-band contact is planned, the user has to put the observation request at least $19 \mathrm{~h}$ before the data acquisition since users are normally not informed about planned radio contacts. However, information on the orbit are publicly available.

In the timeline among others the two observation requests under consideration and subsequent S- and X-band contacts are scheduled (shown in blue in Fig. 1) subject to satellite limitations like power and the predicted orbit. Telecommands (and telemetry) representing this timeline are exchanged with the satellite and involved ground stations are informed about the planned S- and X-band contacts. At the computed times the acquisitions are performed by the satellite. The orbit and attitude data covering an acquisition are received via X-band in the next contact whereas this cannot be assured for all spectral channels of a data acquisition (not the case for request \#1, the case for request \#2). But after $24 \mathrm{~h}$ the whole mass memory is dumped once (and the dump is performed by the first-in-first-out principle). A redump in case of data loss is normally not foreseen. At most, one day after an X-band downlink the corresponding orbit and attitude products are generated and long-term archived. When these products and all spectral channels of a data acquisition are available at the processing facility, valid calibration tables are either already long-term archived or processing of the data is delayed, the long-term archived products each representing a tile of approximately $30 \times 30 \mathrm{~km}^{2}$ will be generated. This is done in at most six days. The specific duration depends on the work load due to the generation of higher-level products to be delivered. In at most four days, the higher-level products to be delivered are generated based on the long-term archived products (request \#1, request \#2, and request \#3).

It is noted, that in particular the monitoring of the complete satellite including its software maintenance, the maintenance of the orbit by orbit maneuvers, the handling of anomalies and nonroutine phases including the reprocessing capabilities of products, the maintenance of calibration and reference tables, as well as a quality control of all the products are not considered in the described routine operations.

The development methodology to establish and operate all these tasks and the design itself are considered. Here, the objective is to illustrate the strengths of the global coherence of the segment-wide approach bringing the different aspects of in particular criticality, novelty, and specificity together and the focus is not on the local details of segment-specific issues. To measure the maturity of the design, its stability across the project phases is examined.

\section{Development Methodology and the Design}

The main purpose of the development methodology is to provide a complete and consistent description of the GS always reflecting its most recent (but also historic) status. Among other tasks, this is important for an efficient configuration management and simplifying the understanding of complexity associated with establishing and operating of large heterogeneous systems, which have to cope with different levels of criticality, novelty, specificity, and to be operated for many years. A logical model of the GS is evolved with its constituents and their relations. This is implemented in a so-called Project Management Support Tool, where information on the recent (but also historic) configurations are always accessible through a web-interface to a relational database management system. By default, each entry has a (meaningful) unique identifier, name, remarks box, and is linked to a person responsible for it.

\subsection{Phases}

The objective of introducing phases and formal milestones (including reviews) is to minimize the economic, scheduling, and technical risks of the project. This is achieved by the identification of clearly defined overall objectives and work content for each phase and the milestones tie together the phases. Thereby, the evolution of agreed baselines is controlled in a traceable manner. 
The establishment and operations of the GS is divided into eight phases with respect to the progress and one additional state.

\subsubsection{Requirements phase}

The requirements phase establishes the requirements (see Fig. 2). At the preliminary requirements review (PRR), the mission requirements are consolidated and the system requirements review (SRR) as the final milestone of this phase, the ground segment requirements are consolidated.

\subsubsection{Design phase}

The design phase establishes the product tree and interfaces (see Fig. 3) including the use cases illustrated in Sec. 1.4. At the preliminary design review (PDR), the first-level decomposition of the GS into subsystems and corresponding requirement specifications are consolidated and at the critical design review (CDR) as final milestone of this phase, the complete decomposition of the GS into its constituents and interface items specifying communications between constituents of the GS are consolidated.

\subsubsection{Technical verification and validation phase}

The technical verification and validation phase establishes the assemblies and test plans, as well as the assemblies and test reports (see Fig. 4) concerning the technical part of the GS, namely testing the facilities. At the technical verification and validation readiness review (TVVRR), the testing activities, or how constituents of the GS are completely and correctly integrated to a GS, are consolidated and at the technical verification and validation review (TVVR) as final milestone of this phase, the technical part of the GS that constituents of the GS are completely and correctly integrated to a GS is accepted.

\subsubsection{Operational validation phase}

The operational validation phase establishes the assemblies and test plans, as well as the assemblies and test reports concerning the operations part of the GS, namely training the operations organizations. At the operational validation readiness review, the training activities are

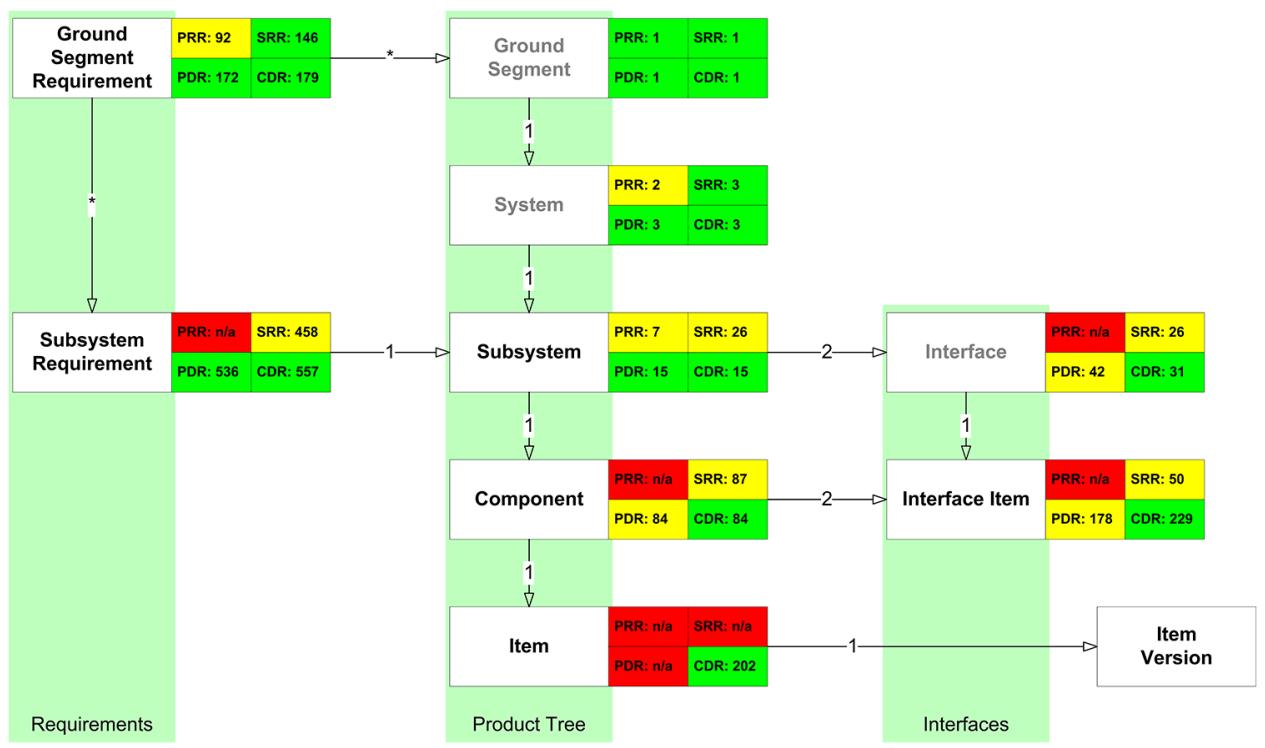

Fig. 3 Design constituents (red: not considered, yellow: draft, green: issue). 


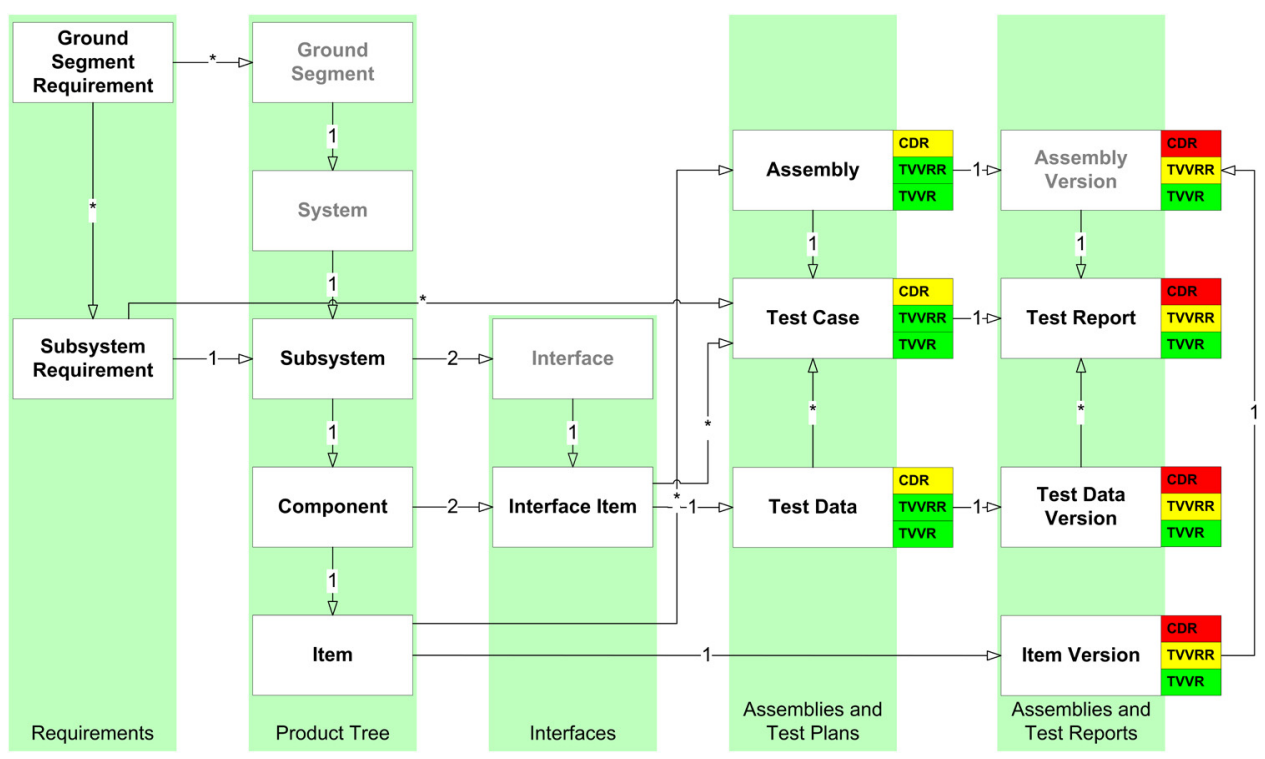

Fig. 4 Test constituents (red: not considered, yellow: draft, green: issue).

consolidated and at the operational readiness review as final milestone of this phase, the operations part of the GS is accepted.

\subsubsection{Phases and state after satellite launch}

The phases and state after satellite launch with their respective objectives are (in chronological order):

- During the launch and early orbit phase, the satellite excluding the instrument is brought into nominal operations.

- In the commissioning phase, the satellite instrument and the GS are installing their nominal operations.

- During the routine phase, satellite and GS are in productive mode. In the de-orbiting phase, the satellite and the GS excluding the data archives are taken out of service.

- The anomaly state is entered when during the routine phase contingency situations concerning the satellite or the GS occur. Resumption to the routine phase is carried out when the contingency situation is resolved.

We focus on the technical part of the GS and in particular on its design, but the methodology for the operations part of the GS is streamlined.

\subsection{Design Constituents}

The evolution of requirements, product tree, and interfaces in the GS is considered more detailed as illustrated in Fig. 3, where for each of the major reviews until the end of the design phase as mentioned in Sec. 2.1 the status of the corresponding group of constituents is indicated together with the cardinality of the group of constituents.

\subsubsection{Requirements}

The development of the GS is driven by the ground segment requirements (GSReq) provided by the Space Agency. The GS is decomposed into a couple of subsystems where each subsystem shall fulfill its set of subsystem requirements (SSReq), which are derived from GSReq. We observe that the ratio GSReq/SSReq is stable at 3.1 for all reviews and in the design phase only minor changes occurred on the requirements both on the number and description. Each SSReq is traced to a subset of GSReq and this is performed such that the functionality of 
the GS as a whole is guaranteed by the set of subsystems resulting in a compliance status (fully compliant, partially compliant, not compliant) for each GSReq. For each subsystem requirement, the subset of verification methods (test, analysis, inspection, review-of-design) is specified. The average number of SSReq traced to GSReq is 5.6 resulting in an average number of 1.8 of GSReq traced to SSReq.

\subsubsection{Product tree}

The previously mentioned subsystems (SS) forming the GS are grouped into systems. At the beginning of the GS development there were only two systems, but the larger one was split such that three systems of approximately equal size resulted. This separation is necessary mainly for management purposes since the SS are assigned to different organizations with different management responsibilities. Therefore, the corresponding box is marked gray. At the beginning of the design phase the number of SS decreased strongly since the four processors, which exist twice, one chain in controlled maintenance and one chain in operations environment, are shifted from SS level by one level down seen from the GS as root since in particular the processors exchange between the environments is thereby logically modeled and the task of chaining the processors is thereby apparently modeled. Each SS is further decomposed into components, which could be either of type facility, modeling the technical part, or operations organization, modeling the operations part. This is the first level where this separation takes place. So, an SS normally consists of both types of components. A component can typically provide a specific functionality on its own. Components (and their constituents) of type operations organization are not counted and not considered here. At the beginning of the design phase, the number of components decreased strongly (taking the mentioned shift into account) since in particular the novelty and specificity of components was considered more strictly that allowed both to pool elements, where only minor changes of the configuration are expected, and to shift the control of elements from the mission-specific environment to the multimission environment. This is detailed in Sec. 2.4. After the beginning of the design phase, only minor changes occurred on the product tree (not only on their cardinality). To allow for a flexible configuration control each component is further decomposed into items, which form the leaves of the balanced tree. This decomposition has to balance flexibility (in items) and dependability (between items). An item can typically not provide a specific functionality on its own. The average number of SSReq traced to SS is 37.1 varying between 17 and 101. We observe that the number of constituents in the product tree at the end of the design phase increases by a factor of approximately 4 , namely between 2.4 and 5.6 from one level to the next. A more detailed investigation illustrates that there do not exist elements with more than ten constituents. For each element of the product tree its functionality, criticality, novelty, specificity, parent, and list of constituents are stated. Each SS is additionally linked to its list of interfaces and requirements, whereas each component is additionally linked to its interface items. For each time, the item version in the operations environment (or empty if not existent) is associated with the item.

\subsubsection{Interfaces}

The previously mentioned interface items indicate communication sequences between two components either of different subsystems or if at least parts of an involved component are exchanged in the GS, such as the processors. The communication in a subsystem is ensured by the verification of the subsystem requirements. Components can provide functionality on their own while items cannot. Furthermore, the linkage on the component level takes the different types of communication protocols for facility and operations organizations into account. Interface items between components of type operations organization are not counted and not considered here. At the middle of the design phase the workflows are harmonized resulting in a strong decrease of the number of interfaces, but also the workflows for anomalies are identified resulting in a strong decrease of the number of interface items. We observe that at the end of the design phase 30 out of 105 SS external plus 1 out of 15 SS internal connections that are possible between subsystems are established. The average number of interface items per 
interface is 7.4, varying between 1 and 33. The average number of interface items per subsystem is 30.5. There exists, on the one hand, one subsystem communicating with nine other subsystems on 150 interface items and on the other hand one subsystem communicating with one other subsystem on one interface items. No other subsystem is connected with more than six subsystems. Of course, there are also interfaces to the both other segments beside the S- and X-band communication and GS internal communications. For each interface item its functionality, involved components, information on initialization, protocol, which is often file transfer protocol (FTP) for data and hypertext transfer protocol (HTTP) for control flows, frequency and size are stated. The interfaces just group the interface items between two subsystems. Therefore, the corresponding box is marked gray.

\subsubsection{Use cases}

The uses cases bring the items and interface items together based on the requirements, namely they define the data flows in particular for the phases and state after satellite launch stated in Sec. 2.1.5. The tasks mentioned in particular in Sec. 1.4 are covered by 18 ground segment-wide use cases.

A relative small change in the cardinality of the design constituents illustrates a high maturity of the design (constituents).

Since the realization of the design has to be tested, the methodology for the test activities of the GS is streamlined.

\subsection{Test Constituents}

The evolution of assemblies and test plans as well as reports in the GS is considered more detailed as illustrated in Fig. 4, where for each of the major reviews until the end of the technical verification and validation phase (see Sec. 2.1) the status of the corresponding group of constituents is indicated (red: not considered, yellow: draft, green: issue).

\subsubsection{Assemblies and test plans}

The items have to be integrated vertically according to the product tree to subsystems. Thereby, it is proven that all subsystem requirements are fulfilled. The interface items have to be verified between two subsystems and validated in the context of use cases, namely for assemblies of subsystems. Thereby, it is proven that the complete GS is ready for usage. This is, roughly speaking, at first done only for the technical part, namely the facilities, and then for the operations part, namely the operations organizations. Compatibility with the space segment is demonstrated with help of the spacecraft simulator and (parts of) the spacecraft itself. The different test cases, even those considering the same interface item, need different types of the instances of interface items, which are represented by test data.

\subsubsection{Assemblies and test reports}

The instantiation of test cases are test reports, namely for each test case and each verified and validated configuration a test report is generated. A configuration is identified by the item and interface item version forming the assembly version of the assembly under consideration. The changes from one version to the next are continuously logged and each product is annotated with this version information containing recursively that of all elements handled the products it is based on. The test data versions support the traceable test case execution.

\subsection{Criticality, Novelty, and Specificity}

The different levels of criticality, novelty, and specificity are drivers for the design of a satellite ground segment. These topics are discussed more detailed in this section. 


\subsubsection{Criticality}

The criticality is identified on component basis since it is the lowest level of the GS, where functionalities are specified (see Ref. 9 for a methodology and Ref. 10 for an example). Analysis is performed based on the designed component functionalities in the context of the use cases. Only single element failures are considered. Each failure mode is evaluated in terms of the worst potential consequences, namely without consideration of compensating provisions, and the associated severity category is assigned. Since in particular the (unmanned) satellite will be completely destroyed during its re-entry before reaching the lower atmosphere, loss of life has not to be considered (risk is avoided). Therefore, a component is classified as critical if a failure mode is identified with a failure consequence leading to loss of mission or permanent degradation of mission. Here, this is specified to loss of data or data not fulfilling the requirements for a not limited amount of time, and comprises past and future mission data. For the likelihood category assignment, the designed compensating provisions are considered. In total, 9 (marked red in Fig. 5) out of 82 components are identified to be critical. Seven affect future mission data, such as collision of the satellite with space debris (risk is accepted) or blinding and thereby damage of the detectors, namely the telescope is pointing to the Sun with open shutter (risk is reduced, e.g., by additionally computing angular difference between telescope and Sun directions and comparing the value with a maximal allowed one during attitude profile calculations). Two affect past mission data, such as loss of the mission data archive (risk is reduced by providing two locally separated libraries applying different storage technologies).

\subsubsection{Novelty}

The novelty is identified on item (and interface item) basis, namely on the lowest level visible to the GS (see Ref. 11 for a methodology and Ref. 12 for an example). Depending in particular on the novelty of an item but also on its criticality, the intensity of the technical verification (and operational validation) is planned. In total, 74 (marked red in Fig. 5) (for example the four processors) out of 202 items are identified to be not reused and 36 are reused (marked green in Fig. 5) (for example the S- and X-band antenna systems). The remaining 92 items are almost equally distributed among reused with minor (marked yellow in Fig. 5) or major (marked orange in Fig. 5) modifications.

\subsubsection{Specificity}

The specificity is identified on item basis. Here, it is classified as mission-specific or multimission on the basis of the lowest level visible to the GS (see Ref. 13 for a methodology and Ref. 14 for an example). A multimission item supports more than one project simultaneously and performs adequate configuration and change control. Such items are under configuration management of the multimission environment, provided that the GS has access to configuration

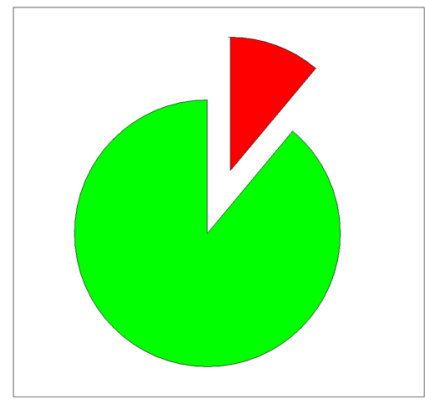

(a)

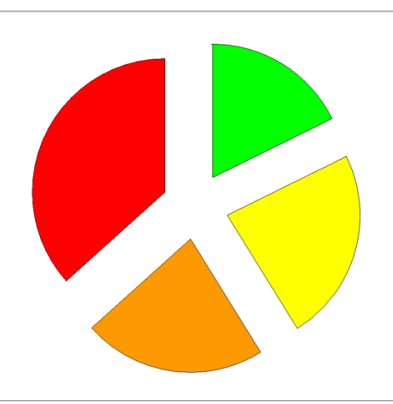

(b)

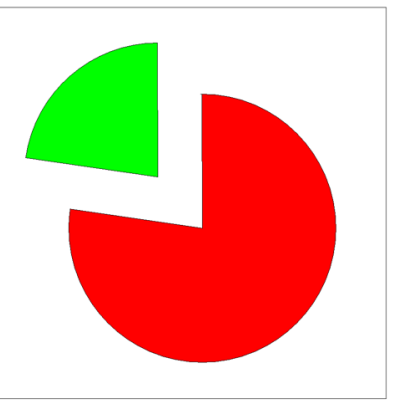

(c)

Fig. 5 (a) Criticality (percent of components; red: critical, green: not critical), (b) novelty (percent of items; red: not reused, orange: reused with major modifications, yellow: reused with minor modification, green: reused), and (c) specificity (percent of items; red: mission-specific, green: multimission). 
documentation. However, changes, which potentially effect the GS have also to be handled according to the change management of the GS and changes have to be cumulatively documented in the change management of the GS in particular prior to technical verification (and operational validation) of the item and on demand, for example, for reviews. In total, 46 (marked green in Fig. 5) out of 202 items are identified to be multimission (for example, the network elements).

\subsection{Two Examples}

The design of two components, namely the product library and the level 2 geo processor that are described in Fig. 6, illustrate the heterogeneity of a satellite ground segment with respect to the aspects mentioned in Secs. 2.2 and 2.4. It illustrates the realization of the mentioned aspects. These topics are discussed in more detail in this section.

\subsubsection{Product library}

The product library stores products for the long-term and provides comprehensive product management functionalities such as product insertion, querying, retrieval, and removal, as well as version and access control. ${ }^{15}$ It is a component of the data and information management system, which consists of, beside the product library, ordering, production, and distributing functionalities including online user services. The product libraries archives beside all uncorrected image data, all orbit and attitude data as well as all calibration and reference tables. The component is classified as critical since the destruction of the product library by disk or operator error leads to loss of mission data. Therefore, two locally separated libraries applying different storage technologies are provided. Since the product library contains various Earth observation products and performs a dedicated configuration and change management, the product library is classified as multimission and is reused with minor modifications since only a limited number of configuration files have to be adapted in order to handle the mentioned data. Hence, the component consists of two items, namely the two libraries, and 78 interface items to handle the mentioned data and control data flows. To illustrate that the requirements can be fulfilled, no simulator and no prototype were created. These were taken from other missions instead, encorporating similar interfaces with a similar amount of data to handle. ${ }^{16}$

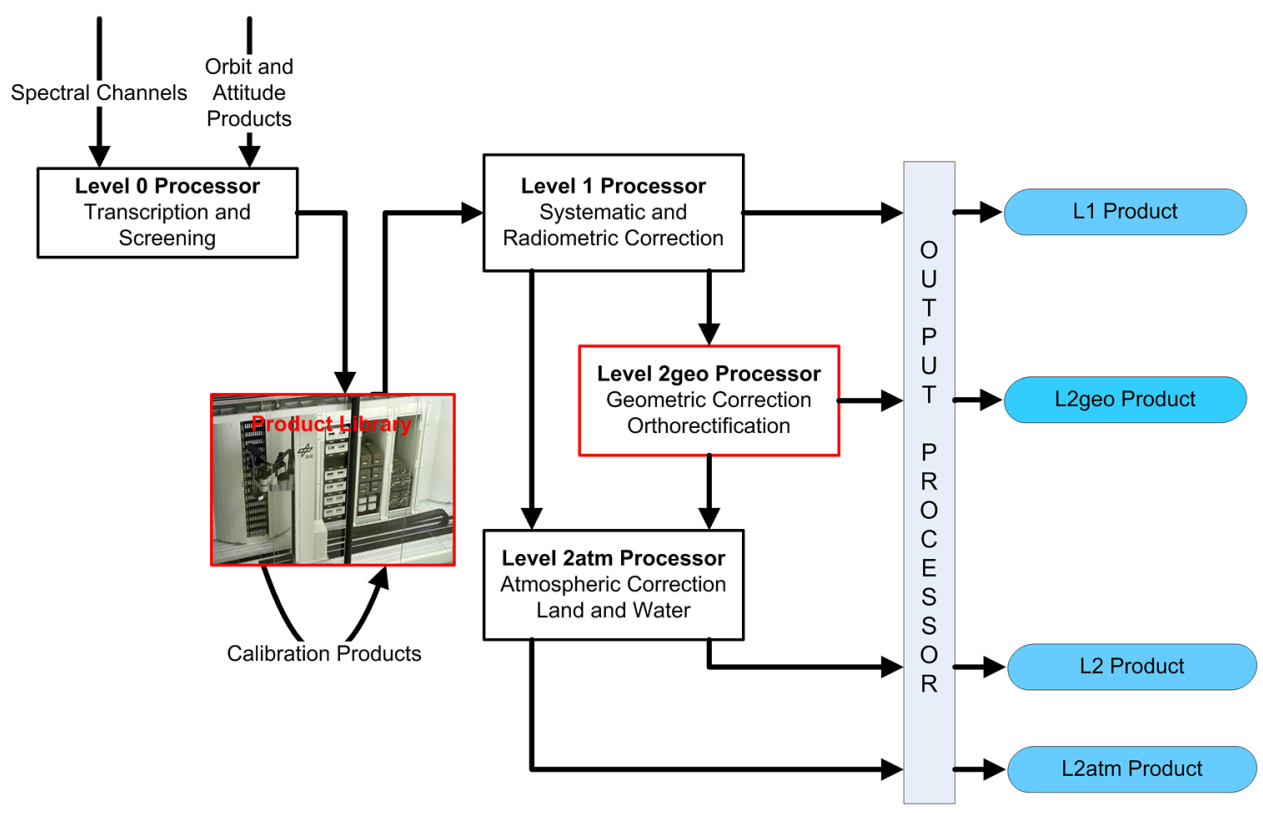

Fig. 6 Product library and level 2geo processor. 


\subsubsection{Level 2 geo processor}

The level 2 geo processor converts radiometrically corrected images to map-accurate forms, namely orthoimages. ${ }^{17}$ This is done by removing:

- sensor distortions based on the interior orientation determined by pre- and in-flight characterizations and calibrations,

- satellite motions based on timing, position, and pointing data determined by measurements of the satellite such as real time clocks, global navigation systems, and star tracker systems and taking into account light aberration and atmospheric refraction, and

- terrain related geometric distortions based on a digital elevation model (DEM).

It is a component of the processor system, which consists of, beside the level 2 geo processor, level 0 , level 1 , and level $2 \mathrm{~atm}$ processors as well as chaining and quality control of these processors. To achieve an absolute geometric error of at most $30 \mathrm{~m}$ image-to-image matching algorithms to global reference images (GRI) are applied. To achieve a relative geometric, namely co-registration, error of at most $6 \mathrm{~m}$ between the two spectrometers not only the absolute geometric but also the geometric error during $86 \mathrm{~ms}$ has to be considered. (See also Sec. 1.3.) The component is classified as noncritical, as mission-specific, and is reused with major modifications. Hence, the component consists of 3 items, namely the level 2 geo processor, the DEM database, and the GRI database, and 0 interface items. It is noted that the items are established by one team and operated by another team, even geographically distributed. The corresponding item exchange procedure is therefore also established as an interface item. To illustrate that the requirements can be fulfilled as detailed in a corresponding algorithms theoretical baseline document both a simulator and a prototype were created. The simulator creates image data geometrically together with all relevant additional satellite data conform to the design of the satellite. ${ }^{18}$ The prototype proves the processors feasibility concerning functionality but also technical budgets like processing time and memory. ${ }^{19}$

\section{Conclusion}

The development methodology and the design of the ground segment for the future remote sensing satellite mission Environmental Mapping and Analysis Program (EnMAP; www.enmap.org) was presented and discussed focussing on criticality, novelty, and specificity aspects of such a highly complex system which is essentially based on software. Because of its heterogeneity, but also that of the team, the focus was less on the local details and more on the advantages of the global coherence of the approach bringing all these aspects together. It was pointed out that beside the stability of the complex design across the project phases in a well-defined methodology, the monitoring of the mentioned aspects are the essential factors for establishing a mature operational satellite ground segment.

\section{Acknowledgments}

Supported by the DLR Space Administration with funds of the German Federal Ministry of Economic Affairs and Technology on the basis of a decision by the German Bundestag (50 EE 0850).

\section{References}

1. F. Pasian et al., "Science ground segment for the ESA Euclid mission," Proc. SPIE 8451, 845104 (2012), http://dx.doi.org/10.1117/12.926026.

2. T. Lock et al., "Product and quality assurance processes and ECSS compliance within a science ground segment using Gaia as an example," Proc. SPIE 8449, 84491D (2012), http://dx.doi.org/10.1117/12.926795.

3. T. Stuffler et al., "The EnMAP hyperspectral imager-an advanced optical payload for future applications in Earth observation programmes," Acta Astronaut. 61(1-6), 115-120 (2007), http://dx.doi.org/10.1016/j.actaastro.2007.01.033. 
4. G. Rossner et al., "EnMAP-Germany's hyperspectral Earth observation mission: outline and objectives of the EnMAP mission," in Proc. EARSeL SIG-IS Workshop, EARSeL, Tel Aviv, Israel (2009).

5. H. Kaufmann et al., "Review of EnMAP scientific potential and preparation phase," in Proc. EARSeL SIG-IS Workshop, EARSeL, Tel Aviv, Israel (2009).

6. S.-N. Haubrock et al., "Surface soil moisture quantification and validation based on hyperspectral data and field measurements," J. Appl. Remote Sens. 2(1), 023552 (2008), http://dx .doi.org/10.1117/1.3059191.

7. B. Sang et al., "The EnMAP hyperspectral imaging spectrometer: instrument concept, calibration, and technologies," Proc. SPIE 7086, 708605 (2009), http://dx.doi.org/10.1117/12 .794870 .

8. T. Storch et al., "On the design of the ground segment for the future hyperspectral satellite mission EnMAP," in Proc. 2010 IEEE Aerospace Conf., pp. 1-11, IEEE Press, Big Sky, Montana (2010).

9. J. Bowen and V. Stavridou, "Safety-critical systems, formal methods and standards," Software Eng. J. 8(4), 189-209 (1993), http://dx.doi.org/10.1049/sej.1993.0025.

10. W. R. Ansorge, "Computers and software in safety-critical applications," Proc. SPIE 4009, 186-196 (2000), http://dx.doi.org/10.1117/12.388388.

11. W. B. Frakes and K. Kang, "Software reuse research: status and future," IEEE Trans. Software Eng. 31(7), 529-536 (2005), http://dx.doi.org/10.1109/TSE.2005.85.

12. J. Marshall, R. Downs, and S. Samadi, "Relevance of software reuse in building advanced scientific data processing systems," Earth Sci. Inf. 3(1), 95-100 (2010), http://dx.doi.org/10 $.1007 / \mathrm{s} 12145-010-0054-3$

13. B. A. Kitchenham et al., "Towards an ontology of software maintenance," J. Software Maint. Res. Pract. 11(6), 365-389 (2002), http://dx.doi.org/10.1002/(SICI)1096-908X (199911/12)11:6<365::AID-SMR200>3.0.CO;2-W.

14. S. Webster, G. Miller, and G. Mayott, "Software as a service approach to sensor simulation software deployment," Proc. SPIE 8403, 84030I (2000), http://dx.doi.org/10.1117/12 .920759 .

15. M. Wolfmüller et al., "Dataflow and workflow organization-The data management for the TerraSAR-X payload ground segment," IEEE Trans. Geosci. Remote Sens. 47(1), 44-50 (2009), http://dx.doi.org/10.1109/TGRS.2008.2003074.

16. S. Buckreuss and B. Schättler, "The TerraSAR-X ground segment," IEEE Trans. Geosci. Remote Sens. 48(2), 623-632 (2010), http://dx.doi.org/10.1109/TGRS.2009.2031432.

17. T. Storch et al., "Processing chain for the future hyperspectral mission EnMAP," in Proc. EARSeL SIG-IS Workshop, EARSeL, Tel Aviv, Israel (2009).

18. P. Schwind et al., "An in-depth simulation of EnMAP geometry," IRSRS J. Photogramm. Remote Sens. 70, 99-106 (2012), http://dx.doi.org/10.1016/j.isprsjprs.2012.03.012.

19. P. Schwind et al., "Processors for ALOS optical data: Deconvolution, DEM generation, orthorectification, and atmospheric correction," IEEE Trans. Geosci. Remote Sens. 47(12), 4074-4082 (2009), http://dx.doi.org/10.1109/TGRS.2009.2015941.

Biographies and photographs of the authors not available. 